\title{
Protée
}

\section{L’ordinateur de Chris Marker. Mélancolie et intermédialité}

\section{Johanne Villeneuve}

Volume 28, numéro 3, 2000

Mélancolie entre les arts

URI : https://id.erudit.org/iderudit/030599ar

DOI : https://doi.org/10.7202/030599ar

Aller au sommaire du numéro

Éditeur(s)

Département des arts et lettres - Université du Québec à Chicoutimi

ISSN

0300-3523 (imprimé)

1708-2307 (numérique)

Découvrir la revue

Citer cet article

Villeneuve, J. (2000). L’ordinateur de Chris Marker. Mélancolie et intermédialité. Protée, 28(3), 7-12. https://doi.org/10.7202/030599ar

\section{Résumé de l'article}

Cet article est une contribution à la définition de l'intermédialité. Dans un premier temps, il décrit les médiations modernes d'après leur caractère paradoxal : le fait que l'effacement du médium soit compris, par exemple, comme le signe même de son efficacité. Dans ce contexte, on peut considérer l'intermédialité comme une certaine dramatisation du paradoxe de la médiation, au sens où l'intermédialité serait la figure de la médiation dans la médiation même. Dans un second temps, cet article se porte vers le film de Chris Marker, Level Five, qu'il désigne comme un locus où cette figure peut être déployée depuis la médiation des morts, la mélancolie et les catastrophes du $\mathrm{XX}^{\mathrm{e}}$ siècle - une mélancolie plus proche néanmoins des anciennes conceptions des humeurs (Aristote) que des ruines romantiques. 


\section{L'O RDIN ATEU R DE CHRIS M ARKER MÉLAN CO LIE ET INTERM ÉD IALITÉ1}
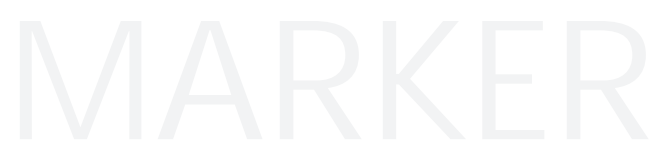

JOHANNE VILLENEU VE

Pour l'écrivain albanais Ismail Kadaré, les premières légendes seraient les premières sculptures, comme autant de «chemins menant de la mort à la vie». Au milieu de la fête dionysiaque et sous l'effet du vin, les sculptures - enveloppes des morts - paraissaient se mouvoir, comme si les morts revenaient à la vie ${ }^{2}$. Cette «vie» advient toutefois sous le mode de la «revenance», car c'est bien le mort qui revient parmi les vivants, à travers la légende, c'est-à-dire dans la matérialité même de la pierre; simplement, la pierre se meut, agite la mort en elle comme la vie dans sa chrysalide. La légende, suggérait Kadaré, espace de "voiles et de brouillards", fournit donc "une lecture fantomatique du monde», pour le meilleur et pour le pire, car les statues de Staline appartiennent aussi à la légende. La chrysalide auratique enferme et produit l'espérance d'un monde meilleur; en elle se préparent autant de désastres que de merveilles.

Puisque le titre de cet article annonce l'ordinateur de Chris Marker, ceux qui ont vu le film Level Five auront sans doute perçu, dans ce bref prélude consacré à la légende et à sa chrysalide, une affinité avec le film: assise devant un ordinateur, une femme au nom auratique (Laura), filmée plein cadre, se livre à un jeu vidéo terrible: reconstituer la «bataille» oubliée d'Okinawa - événement fantôme de la fin de la Deuxième Guerre mondiale. Ce jeu est aussi ce qui reste à Laura d'un homme qu'elle a aimé et qui est mort - perte qui s'emmêle sur la spirale du temps si chère à Marker, et dont la profonde mélancolie rejoint une autre mémoire: les morts d'Okinawa, les suicidés solitaires et collectifs, les archives du siècle, la falsification de l'histoire et les témoignages des survivants.

Laura est si proche du spectateur - immense visage et immense bouche sur l'écran immense - qu'elle fait justement écran et renvoie à l'impossible communication, à ce qui la sépare de l'autre côté, à savoir le côté des morts, mais aussi le côté où nous sommes et qui la désigne, elle, en un miroir insolite, «fantôme». Intervient la voix de Marker lui-même, spectrale, parlant de sa rencontre avec Laura au passé, croisant sa voix à la sienne, pénétrant dans la zone où circulent les images. Leur rencontre n'a pourtant jamais lieu, elle se remplit de cette distance qui les lie et les sépare tout à la fois, cette distance et cette proximité 
qui nous lient et nous séparent aussi d'un visage géant et d'une voix d'outre-lieu dont la présence amplifiée comble nos oreilles.

Cette distance, on doit à la fois la rompre et la mesurer, comme autrefois la fête dionysiaque permettait de rompre et de mesurer la distance avec les morts et leur monumentalité. Ce double geste, qui consiste à mesurer la distance qui nous sépare de ce qui est et à rompre tout à la fois cette distance, serait, on l'a vu, le propre de la légende.

J'aimerais reprendre la même formule pour décrire ce qui dépasse la seule légende mais qui en constitue néanmoins l'opération fondatrice: la médiation. Une allégorie peut en exemplifier les traits: «l'antique messager» parcourant à pied ou à cheval des contrées éloignées: son périple se mesure en jours, en mois, et en chemin parcouru. En portant son message d'une contrée à une autre, le messager scelle un lien dans l'espace et dans le temps, mais sa course n'est-elle pas aussi la mesure de la distance qui sépare un point de départ d'un point d'arrivée? Ainsi croit-on pouvoir en dire autant de toute médiation. Distance et proximité, absence et présence, médiateté et immédiateté semblent se confondre dès qu'il s'agit de penser ce qu'il en est des médias. Ce que j'appellerai ici le "paradoxe de la médiation" tient à l'impression de ce que plus le médium est efficace, plus il tend à disparaître: le cavalier le plus rapide n'est-il pas celui qui, filant à grande allure, se laisse à peine saisir sur la ligne de l'horizon? Ainsi, l'effacement du médium paraît garant de la médiation. Pourtant, nous le savons tous, jamais la diligence du messager n'arrivera à le faire disparaittre complètement; dans l'effacement même, voyant le coursier s'abolir dans sa course, je garde une impression: celle de la course même.

Dans le contexte médiatique, l'impression d'effacement du médium s'accompagne d'une autre impression tout aussi importante: l'effet que peuvent avoir, par exemple, les images dans le mystère de leur matérialité, comme tout à l'heure la sculpture ancienne retenait enfermée dans la pierre la noce entre le vivant et le mort. La confusion qui guette le spectateur de cinéma n'est pas une confusion entre le réel et la fiction, entre ceci et cela, mais au cœur même de la médiation, comme «un brouillard» dans la chrysalide, comme le mort rendu vivant dans la matérialité de la sculpture. Si l'époque actuelle, chargée d'hypermédialité, tend à ignorer le médium, cela paraît tenir de la médiation elle-même et de son paradoxe: plus on accorde de l'importance à la médiation, plus on en célèbre la capacité à s'abolir dans le mouvement.

Ce qui se laisse décrire ici en termes de paradoxe n'est pourtant pas compris de même dans les cultures traditionnelles d'où sont issues les premières médiations entre les vivants et les morts. Non pas parce que les gens n'accordent aucune valeur à la médiation et ignorent ce qu'elle est, mais bien parce qu'elle est si bien intégrée à leur expérience qu'elle désigne ce qu'est à leurs yeux la vie elle-même. Pour l'homme traditionnel, il n'y a pas de réalité ni de fiction à transmettre comme un objet de connaissance. La médiation palpite au cœur de la mémoire collective, toujours déjà là, dans les récits scandés depuis la nuit des temps comme dans le geste quotidien. Les morts, les Anciens, les Esprits n'ont jamais quitté les vivants. Non pas au sens où ils ont toujours été proches, mais au sens où le lointain n'a jamais cessé de séjourner chez les vivants. Cette médiation, au sens traditionnel, nous le comprenons mieux maintenant, ne saurait être vécue comme une confusion entre la réalité et sa représentation, ni davantage comme un paradoxe, mais elle ouvre néanmoins l'espace d'un «mélange» entre le vivant et le mort - une ambiance où le proche et le lointain diffèrent et s'allient, mais ne se déchirent pas. L'oralité y fait pour beaucoup. Les morts traversent l'espacetemps dans, par et à travers la voix shamanique, parce que la voix humaine ne connaît pas de différence entre le dans, le sur, le par et le à travers. Entrant dans la voix humaine, les morts entrent dans le mouvement de la vie; la voix n'est ni leur support, ni leur relais; elle est l'avènement du lointain.

C'est avec la modernité que le paradoxe de la médiation s'entend précisément comme paradoxe. Les raisons de ce revirement sont nombreuses et 
complexes; je me contenterai, en suivant le filon de la matérialité du médium, d'admettre que cette dramatisation de la médiation (qui est bien sûr une dramatisation de la relation entre la matérialité du médium et son efficacité) tient au passage de l'oralité à l'écriture. Le livre et à plus forte raison sa reproductibilité confèrent à l'expérience et au savoir, à la mémoire et au passé, une nouvelle autonomie en leur permettant de circuler sans le secours de la voix et du corps. Cette autonomie a un impact considérable: elle cristallise le rapport entre l'effacement du médium et sa matérialité; elle trouble le rapport avec les morts, le passé, la mémoire, en accentuant des écarts; elle redouble le rapport entre distance et proximité. C'est comme si le messager rencontrait au milieu de sa course la figure de sa course même. Cette figure, je l'appellerai «intermédialité». C'est à cette dramatisation du paradoxe de la médiation, à son vertige, que j'associe l'intermédialité: la figure de la médiation dans la médiation même, cet «inter» de «l'inter».

La question de l'intermédialité - et en l'occurrence celle que toute interaction actuelle entre les arts devrait susciter - est alors la suivante: selon quelle médiation les différents médias entrent-ils en interaction et en conflit?

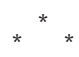

La question qui agite le film Level Five de Chris Marker me semble déployer justement cette figure de l'intermédialité. Avec ce qu'il est courant d'appeler de nos jours les «médias de l'image», la médiatisation des morts est excessive, tant il nous est donné à voir du cadavre; mais la médiation des morts s'est perdue et ne subsiste plus qu'en de rares épiphanies dont Level Five présente encore la possibilité, parce qu'il met en tension profonde la figure de l'intermédialité avec cette mémoire si lointaine et si proche qu'est la légende. Mais quelle est donc la légende de notre siècle?

Le spectateur partage avec Laura la même inexpérience de la guerre, le sentiment de l'oubli sans cesse ramené à l'expérience intime du deuil. Parlant de ce qu'elle a perdu, Laura nous renvoie en fait à l'aura de sa propre disparition sur laquelle d'ailleurs le film s'achève, non sans rappeler combien la mémoire éclôt souvent chez Marker d'un visage de femme toujours déjà du côté des morts, parmi les masques. Survient alors, au sens plein du terme, une extraordinaire coïncidence entre le vivant et le mort, mais alors même qu'apparaît leur impossible rencontre. Laura a perdu l'amour de sa vie et ne le rencontrera plus qu'à travers les fragments d'un journal électronique, inachevé; Chris Marker ne rencontrera plus Laura que sur l'écran de son film, sous la forme de l'absence. Le présent des choses nous est rendu comme depuis le futur, dans la dimension de leur passé.

Le jeu de l'ordinateur n'a rien de léger. Le ludique puise dans l'enfance une certaine gravité, cette faculté de concentration propre à l'enfant qui joue avec l'assurance d'être pour une fois invincible devant la mort et devant le temps. Dans Level Five, le jeu vidéo comporte la même gravité, mais l'espace du jeu s'ouvre entièrement à la dimension de la mémoire et de l'avenir, du souvenir et de l'espérance ${ }^{3}$. Ce jeu n'est autre que le jeu de la "pensée» qui se cherche dans les dédales bruyants d'un siècle hanté par les cataclysmes et les survivances. Les fantômes d'Okinawa contiennent - au sens d'endiguer et de renfermer- les images du siècle, surdimensionnelles, ondoyantes et insondables, ces images et ces compositions multimédiatiques qui éloignent la femme de l'homme aimé, le vivant du mort, l'adulte de sa propre enfance, l'être contemporain del'être du passé: images qui sont autant de mélanges de paysages et de visages et qui pourtant, comme le disait déjà le narrateur du film Sans Soleil, «ne peuvent être liées». Comment alors faire apparaittre le sens?

Pensons à un autre jeu: Beckett. Théodor Adorno considérait que la «seule compréhension possible» de Fin de partie de Samuel Beckett était la «compréhension de son incompréhensibilité». Au lendemain de la Seconde Guerre, écrivait Adorno, tout était détruit, jusqu'à la résurrection de la culture; 
l'humanité était devenue «végétative», rampant parmi les ruines, «après des événements auxquels même les survivants n'arrivaient pas à survivre» ${ }^{4}$. Depuis son travail avec Alain Resnais dans Nuit et Brouillard, Marker n'a cessé de rappeler et d'annoncer la survivance. Assis aux commandes de la console mémorielle du siècle, le sujet moderne casqué, bardé de technologies, hypermédiatique, semble abandonné à des images qui le dépassent et qui sont pourtant les siennes. L'intermédialité touche à l'éthique, car le vertige qu'elle dessine est aussi celui de la déréliction - la console de Laura est une consolation. Pour l'art, il ne s'agit plus d'interroger les «contenus de l'expérience" par le moyen d'un médium, mais de comprendre les équivoques d'une médiation devenue médiatisation - notre voile et notre brouillard, pour paraphraser Kadaré. Toute expérience a intégré aujourd'hui, jusque dans les rêves, la cinématique des images. Elle l'a intégrée, mais en un vertige, pour le meilleur et pour le pire. Au début du siècle, avec Vertov, la mobilité de l'homme-à-la-caméra pouvait encore être célébrée, à la fois comme l'avènement de la technique et la technique de l'événement; la lentille de Marker exprime une lumineuse tautologie de l'image: la vie médiatisée par la vie elle-même, les images médiatisées par les images. Mais ce que produit cette tautologie, c'est la reconnaissance de la fragilité de l'œil, et non l'efficience de la lentille. La matérialité du monde subit ainsi le trouble d'un regard singulier et s'ouvre à la revenance: il faut s'efforcer de tracer l'effacement des signes, de montrer la disparition des rituels et l'insondabilité des visages, de dessiner les épures du monde qui seront lavées par le temps. Cette gratuité - ce jeu grave - (ce sacrifice, dirait Tarkovski) est le tribut à payer au regard du siècle et la seule condition de l'avenir.

L'intermédialité se fait donc mélancolie. Mais qu'est-ce que la mélancolie? Il faut retourner à un texte attribué de manière incertaine à Aristote, et qui voyagea dans le temps avec une extraordinaire persistance. Le "problème XXX", parfois intitulé "L’homme de génie et la mélancolie" ${ }^{5}$, poursuit en effet une étude amorcée dans De l'âme d'Aristote et achève de tirer la mélancolie hors de la sphère de la maladie pour la désigner comme une condition de la créativité poétique - une poiêsis que j'appellerais aujourd'hui «la pensée». Rappelons que c'est dans De anima qu'Aristote développe sa théorie de l'imagination (phantasia). Il y est donc question des images et de leur mouvement. Selon la même tradition, Rufus d'Ephèse, penseur de la mélancolie, soutenait que ceux qui sont intelligents tombent dans la mélancolie "parce qu'ils sont rapides de mouvements, qu'ils ont beaucoup de facilité à anticiper (praemeditation) et à imaginer (imaginatio)" 6 . On sait par ailleurs que c'est aussi à ces images que l'on doit l'art de l'allégorie et, plus précisément, l'art de la mémoire - cette non moins célèbre mnémotechnique capable de «tirer de loin" les souvenirs grâce aux images. Cette expression «tirer de loin» n'est pas, de ma part, placée ici innocemment. Elle se trouve convoquée dans la description que fait Aristote du mélancolique, plus précisément dans un des traités d'histoire naturelle, celui qui concerne la divination dans le sommeil - sujet tout aussi cher à Chris Marker si l'on se souvient bien de La Jetée et de Sans Soleil. Aristote y précise la nature de la mélancolie en des termes de prime abord surprenants:

Quant aux mélancoliques, à cause de la [force] de leurs sensations, ils atteignent facilement le but, comme s'ils tiraient de loin; et à cause de leur mobilité, ils imaginent rapidement ce qui va suivre [...] les mélancoliques enchaînent les faits les uns aux autres en poussant en avant. ${ }^{7}$

Pour peu nous aurions là une description du cinéma. Mais c'est surtout la métaphore de l'archer qui attire mon attention. Le mélancolique qui atteint son but, "comme s'il tirait de loin", grâce à la force de ses sensations est une image, sans doute éloignée de notre monde où l'on ne tire plus qu'avec des armes qui ne demandent aucune force physique, mais combien prégnante dans le monde d'Aristote. Replacée dans ce contexte, l'image apparait incongrue: comme le remarquait Jackie Pigeaud, spécialiste d'Aristote, [...] « il faut tirer loin pour toucher juste » n'est pas une loi de la balistique, mais elle peut bien être une loi de la Poétique. [...] Il 
n'existe pas de point de vue d'où saisir le paysage, d'où percevoir la cible, d'où la désigner. Il faut tirer, et pour cela d'abord avoir la force de le faire. ${ }^{8}$

Voilà bien une théorie de la médiation et qui résout par-dessus le marché le paradoxe de tout à l'heure. Car passer de l'allégorie du messager à celle de l'archer me permet de saisir l'élément qui me manquait jusqu'ici: la "force». Or cette force est-elle celle de l'archer ou une violence qui l'agite? Aristote semble se contredire en décrivant ailleurs le mélancolique comme un homme fragile parce qu'instable, d'où le risque de la folie et la démultiplication de ses comportements. Comment donc l'archer peut-il faire preuve de force tout en demeurant fragile? Il stipule également dans ses traités que le mélancolique "tire partout», dans de multiples directions. Comment peut-on dire alors qu'il touche juste? Le cinéma de Marker en offre la réponse. Car ne cessant de viser, les médias de l'image peuvent en effet faire voir combien toute image est mouvement, parce que toute image échappe dans le temps au moment même où se présente son «ici-maintenant». Ce qu'un film comme La Jetée arrivait justement à montrer, parce que tourné presque entièrement en plans fixes ${ }^{9}$, c'est ce mouvement qui, bien au-delà du montage, se «tient", de toutes ses forces pourrionsnous dire, au creux de l'image, et qui rappelle que nous sommes toujours dans le temps, et pour le dire comme le narrateur de La Jetée, que nous «n'échappons pas au temps». L'image seule engage inchoativement à la narrativité et appelle le montage comme les restes demandent à être mis en récit, et non le montage qui impérieusement s'imposerait comme seul récit.

Le mélancolique, pour Aristote, n'est pas encore l'homme des ruines, mais l'homme des «résidus». Pour concevoir le mélancolique comme l'homme des ruines, il faut avoir pleinement intériorisé la logique de l'écriture pour laquelle la mémoire est signes. L'homme des «résidus» est à cheval entre l'oralité et l'écriture, comme la pensée aristotélicienne est ellemême entre une tradition orale encore très forte et la raison systématisante. J'en veux justement pour preuve sa conception de l'imagination dont la source est le corps. C'est dans les troubles du corps et le mélange de ses humeurs que se définissent la notion d'imagination et la figure du mélancolique. À la différence des ruines, les résidus constituent un mélange; à proprement parler il s'agit de ce qui n'est pas cuit par la digestion. Mais la matérialité de ces résidus n'est pas divisible; il n'y a pas d'étalon possible là où le mouvement des humeurs s'apparente au mouvement de l'eau et de l'air. Le vent et le liquide composent le résiduel. C'est à la force de ce mouvement que fait référence la métaphore de l'archer dans la conception de la mélancolie. Si la médiation se conçoit maintenant comme «force» et non plus simplement comme passage, si elle se conçoit comme «mouvement» non seulement dans l'espace et dans le temps, mais aussi et surtout dans le corps, alors la médiation est une «intensité» et non plus seulement un "pont» entre deux rives.

Le modèle dont dépend Aristote, sans avoir même besoin de le reconnaître, est celui du son, provenant bien évidemment de la tradition orale.

Dans sa célèbre étude Orality and Literacy, Walter Ong rappelle la «force» du mot dans les cultures orales: le son ne peut être sans l'utilisation de la force. Il est fondamentalement dynamique. Dans une culture pour laquelle toute la mémoire du monde repose sur le son, le caractère éphémère du mot prononcé, et aussitôt dissous, n'a rien de paradoxal. Car on reconnaît à la «force» du son le pouvoir d'agir et de perpétuer la mémoire commune. Pour la culture orale, le son n'est pas divisible en signes, mais entièrement coulé dans la médiation, à la fois comme évanescence et comme force d'avènement.

Si le mélancolique est à la fois fragile et fort pour Aristote, c'est bien parce que, sur le modèle du son, sa force et sa fragilité concordent à travers l'éphémère (ce qui se perd) et l'impulsion de "tirer». Il ne faut pas retenir de tout ceci que Chris Marker est un grand mélancolique. Level Five donne à voir combien la médiation qui incombe à l'art aujourd'hui tient de la mélancolie, c'est-à-dire de la force et de la fragilité. 
Cette force et cette fragilité où s'intensifie la revenance du passé et des morts. Prenons une image de Sans Soleil appelée «image de bonheur»: trois enfants filmés en 1965 en Islande sur un lieu qui sera englouti cinq ans plus tard sous la lave d'un volcan. Le narrateur nous dit en voix off que c'est là une image de bonheur. Les trois enfants marchent un peu, s'immobilisent dans le cadre, frêles, solidaires et timides, au milieu d'un paysage immense, incompréhensible. L'image tremble. La pensée glisse dans le souvenir perdu mais toujours retrouvé d'une image, tandis que le regard affronte le vent, «tient» sa visée, secoué par la fragilité des êtres.

La médiation telle que la pratiquaient les cultures orales, le paradoxe de la médiation tel que le dramatisent les cultures de la «littéracité» hantent le mouvement des images au cinéma et imposent encore dans l'ordinateur de Chris Marker la figure de l'intermédialité. Les multiples visées de la mélancolie s'éprennent d'une intensité telle que s'agitent à l'horizon de la pensée les vestiges de l'imagination, de l'oralité et de l'écriture. Si, comme le suggère Gadamer à l'instar de Heidegger, l'œuvre d'art n'est pas un objet mais un événement qui «nous assène un coup, $[\ldots]$ nous renverse en instituant un monde propre dans lequel nous sommes aspirés " ${ }^{10}$, la notion de séjour dans l'œuvre d'art, comme «forme temporelle de l'expérience artistique " ${ }^{11}$, ne convient pas parfaitement à l'événement cinématographique qui institue, non pas tant un "monde propre», une «œuvre-ensemble» comme dirait Gadamer, mais une traversée de la médiation elle-même et de ses paradoxes.

\section{N O TES}

1. Cette recherche a été rendue possible grâce aux fonds accordés par le Conseil de Recherche en Sciences Humaines du Canada.

2. I. Kadaré, 1995: 71.

3. Ironiquement, le cédérom Immemory (1998) créé par Marker n'arrive pas à rendre avec autant de force et de gravité ludique ce que Level Five arrive à rendre. Plus lent et plus limité sur le plan cinématique, le cédérom ne produit aucune catharsis, ce que le film arrive à faire non sans transformer l'ordinateur de Marker en un formidable dispositif de pensée et de narration. Le film intègre l'ordinateur, le propulse au-delà de ses propres capacités, vers la légende ancienne justement, comme à rebours de la technologie, mais par la technologie néanmoins. Le cédérom n’intègre pas les films de Marker, il en évoque simplement le souvenir, comme des images trouvées dans un album de famille.

4. Adorno, 1982: 122 ("Versuch, das Endspiel zu verstehen», dans Noten zur Literatur II, Frankfurt am Main, 1961).

5. Le parcours de ce texte attribué à Aristote est immense. Il est cité dans quantité d'ouvrages depuis la Renaissance jusqu'aux XVIIe et XVIII ${ }^{e}$ où il trouve un succès considérable, jusque dans la psychiatrie du XIX ${ }^{\mathrm{e}}$ siècle (Moreau). L'idée selon laquelle le mélancolique se retire du monde (taciturne et solitaire) et recherche les lieux déserts tient à l'influence des Lettres du Pseudo-Hippocrate datant de la seconde moitié du premier siècle avant J.-C. Les lettres portent surtout sur Démocrite qui s'est retiré dans la nature, "dissèque des animaux et se rit de tout ». Introduction de J. Pigeaud, dans L'Homme de génie et la mélancolie d'Aristote.

6. Ibid., p. 63.

7. Aristote, 1965 [464a]: 92.

8. Pigeaud, 1988: 52-53.

9. La Jetée présente en effet des photographies filmées sur pellicule et constitue, en ce sens, un "roman-photo", tel que l'indique Marker luimême au début de son film. Pourtant, la présentation de ces images photographiques a pour effet de mettre à distance l'image et de la faire voir comme une archive appartenant à cette mémoire que cherche très précisément à nous raconter le film. Or le seul plan non fixe du film, et qui ne tient pas du médium photographique, montre une femme ouvrant les yeux, stigmatisant ainsi le moment utopique, unique et pourtant en mouvement, à la recherche duquel l'ensemble archivistique, cet amalgame photographique de la mélancolie, engage. L'œil qui s'ouvre coïncide ici avec l'ouverture même de l'image cinématographique: une seule image qui surgit dans les dédales et parmi les ruines photographiques, mais qui fait voir combien les autres se meuvent déjà en elle, dans leur fixité même, précisément parce qu'elles sont arrachées à quelque chose, constituées par le manque et par l'oubli.

10. H.-G. Gadamer, 1998: 91.

11. Ibid.

\section{RÉFÉREN CES BIBLIO G RAPHIQ U ES}

AdORNO, T. [1982]: "Trying to Understand Endgame», New German

Critique, University of Wisconsin-Milwaukee, $\mathrm{n}^{\circ} 26$.

ARISTOTE [1988] : L'Homme de génie et la mélancolie, Paris, Rivages; [1980]: De l'âme, Paris, Société d'édition «Les Belles Lettres";

[1965]: Petits traités d'histoire naturelle, Paris, Société d'édition "Les Belles Lettres ", [464a].

Gadamer, H.-G. [1995]: Herméneutique. Esthétique. Philosophie pratique, Paris, Fidès, 1998.

KADARÉ, I. [1995] : La Légende des légendes, Paris, Flammarion. ONG, W.J. [1983]: Orality and Literacy, London \& New York, Methuen. PIgEAUD, J. [1988]: «Introduction", dans Aristote, L'Homme de génie et la mélancolie.

\section{FILMOG RAPH IE ET CÉD ÉRO M}

MARKER, C. [1998]: Immemory (cédérom), Paris, Centre national d'art et de culture Georges-Pompidou;

[1997]: Level Five, Paris, Les Films de l'Astrophore, Argos Films, La Sept Cinéma ;

[1982]: Sans Soleil, Paris, Argos Film;

[1962]: La Jetée, Paris, Argos. 